\title{
Long-lived electroweak-singlet colored scalars
}

\author{
Christian T. Preuss $\odot^{*}$ and German Valencia $\odot^{\dagger}$ \\ School of Physics and Astronomy, Monash University, \\ Wellington Road, Clayton VIC-3800, Australia
}

(Received 8 September 2021; accepted 4 November 2021; published 30 November 2021)

\begin{abstract}
There has been much recent interest in long-lived massive particles at the LHC, understood as those with lifetimes between tens of micrometers and several meters. In this context, we consider the possibility of long-lived electroweak singlet scalars charged under the color SU(3) with masses near a TeV. The shortest lifetime of interest is already longer than typical hadronization scales. These exotic new particles would therefore appear as color-singlet bound states of the new scalars with quarks and gluons, and it is their color charge that prevents them from decaying. In particular, we consider color representations consistent with maintaining asymptotic freedom, those with dimensionality $d_{R} \leq 15$. We find that only the octets can decay, and they do so into multijet final states through the two-gluon channel. The other representations are stable and form fractionally charged color singlets, with the decuplet being the only one that can form electrically neutral color singlets.
\end{abstract}

DOI: 10.1103/PhysRevD.104.095030

\section{INTRODUCTION}

Recently, there has been renewed interest in the study of long-lived particles at the LHC, what is dubbed the lifetime frontier. In the context of LHC studies, the interesting lifetime range lies between tens of micrometers and several meters. Recent reviews that highlight different theoretical scenarios that may give rise to such long-lived particles and the experimental strategies to search for them are, for example, Refs. [1-3].

One intriguing possibility consists of long-lived scalars charged under the color group. Due to color confinement, such particles would hadronize before decaying (if they decay at all) and show up in the detectors as exotic hadrons. Examples of these kinds of particles, which have been studied at length in the literature, are the so-called $R$ hadrons [4]. In this case, gluinos or squarks are pair produced and are either long-lived or stable due to $R$ parity. Other scenarios have also been considered for the case of color triplets $[5,6]$. For the electroweak singlet scalars we consider here, it is their color charge that prevents them from decaying, as will be outlined below.

We present a bottom-up simplified model in which we extend the standard model (SM) with a set of electroweak

\footnotetext{
*Christian.Preuss@ monash.edu
}

†erman.Valencia@monash.edu

Published by the American Physical Society under the terms of the Creative Commons Attribution 4.0 International license. Further distribution of this work must maintain attribution to the author(s) and the published article's title, journal citation, and DOI. Funded by SCOAP. singlet scalars in a representation $R$ of the color $\mathrm{SU}(3)$. These objects would then be copiously pair produced by gluon fusion at the LHC if their masses are below the order of a TeV. Being electroweak singlets, their exotic color structure can prevent them from decaying, and color thus plays the role of $R$ parity in making these particles stable. Carrying a color charge, these hypothetical scalars would also combine with quarks and gluons to form color singlets on typical hadronization time scales. Experimental searches would thus mimic the strategies used for $R$ hadrons.

We find that the requirement of maintaining asymptotic freedom restricts the possible $\mathrm{SU}(3)$ representations to $R=\mathbf{3}, \mathbf{6}, \mathbf{8}, \mathbf{1 0}, \mathbf{1 5}, \mathbf{1 5}^{\prime}$. With masses below $\sim 1 \mathrm{TeV}$, their production cross sections would be in the $\sim 0.1-1000 \mathrm{pb}$ range, and they can potentially introduce significant corrections to the Higgs decay which then serves to constrain them. We find that the octet is the only one of these examples that could decay into two gluons and for most of parameter space, would be short-lived. The others are stable and would hadronize into fractionally charged color singlets with the exception of the color decuplet, which can form an electrically neutral exotic hadron.

This manuscript is structured as follows. In Sec. II, we outline the extension of the SM towards including the new colored scalars based on arguments of conserving asymptotic freedom. Section III focuses on the scalar potential of the new particles. Section IV then discusses all possible decay modes at leading-order (LO) and next-to-leading order (NLO). Before concluding in Sec. VII, we briefly discuss implications on hadrons created by such new colored scalars in Sec. VI. 


\section{COUPLINGS TO GLUONS}

We begin by considering those model-independent couplings that depend only on the color representation of the scalars and that determine their tree-level couplings to gluons. For a complex electroweak-singlet scalar field $S$ transforming with respect to an irreducible representation $R$ of SU(3), its interaction with the gluon field $A^{\mu}$ is dictated by the covariant derivative in the quadratic part of the Lagrangian,

$\mathcal{L}=\left[\left(\partial_{\mu}+\mathrm{i} g_{\mathrm{s}} A_{\mu}^{A} T_{R}^{A}\right) S\right]^{\dagger}\left(\partial^{\mu}+\mathrm{i} g_{\mathrm{s}} A^{A, \mu} T_{R}^{A}\right) S-m_{S}^{2} S^{\dagger} S$.

The coupling strength is the usual strong coupling $g_{\mathrm{s}}$ and in writing eq. (1) we have omitted explicit color indices. For real representations of $\mathrm{SU}(3)$, the scalar fields will be real and an additional factor of $1 / 2$ is required.

\section{A. Asymptotic freedom}

The SU(3) representations that we consider are first restricted by requiring the model to maintain asymptotic freedom at scales above the scalar masses. To this end, we recall the well-known QCD one-loop beta function, to which vector, fermion, and scalar fields charged under the $\mathrm{SU}(3)$ contribute as

$$
\beta_{g}=-\frac{g_{\mathrm{s}}^{3}}{16 \pi^{2}}\left(\frac{11}{3} t_{2}(V)-\frac{4}{3} n_{\mathrm{F}} t_{2}(F)-\frac{1}{3} t_{2}(S)\right) .
$$

Here, $t_{2}(V), t_{2}(F)$, and $t_{2}(S)$ denote the Dynkin index of the representation in which the different fields transform, respectively. For the SM augmented by complex scalars in representations with dimension up to 15, Eq. (2) takes the form,

$$
\begin{aligned}
\beta_{g}= & -\frac{g_{\mathrm{s}}^{3}}{16 \pi^{2}}\left(11-\frac{2}{3} n_{\mathrm{F}}-\frac{1}{6} n_{3}-\frac{5}{6} n_{6}\right. \\
& \left.-n_{8}-\frac{5}{2} n_{10}-\frac{10}{3} n_{15}-\frac{35}{6} n_{15^{\prime}}\right) .
\end{aligned}
$$

In what follows, we will only allow one scalar multiplet at a time, and this implies that asymptotic freedom holds as long as ${ }^{1}$

$$
t_{2}(S)<21
$$

All possible multiplets satisfying Eq. (4) are collected in Table I, and their corresponding pair-production cross section at the LHC is plotted in Fig. 2. The lowest-dimensional representation to fail Eq. (4) is $(3,1)$ for which $t_{2}(\mathbf{2 4})=25$.

\section{B. Production via gluon fusion at the LHC}

Equation (1) determines the scalar pair-production cross section from gluon fusion that proceeds at LO through the diagrams in Fig. 1. For complex scalars, it is given in terms of the quadratic Casimir of the representation, $c_{2}(R)$, by [9]

$$
\frac{\mathrm{d} \sigma}{\mathrm{d} t}=\frac{2 \pi \alpha_{\mathrm{s}}^{2}}{s^{2}} \frac{c_{2}(R) \operatorname{dim} R}{(\operatorname{dim} A)^{2}}\left[\left(c_{2}(R)-\frac{1}{4} c_{2}(A)\right)+\frac{c_{2}(A)}{4} \frac{(u-t)^{2}}{s^{2}}\right] \times\left[1+\frac{2 m_{S}^{2} t}{\left(m_{S}^{2}-t\right)^{2}}+\frac{2 m_{S}^{2} u}{\left(m_{S}^{2}-u\right)^{2}}+\frac{4 m_{S}^{4}}{\left(m_{S}^{2}-t\right)\left(m_{S}^{2}-u\right)}\right] .
$$

The terms depending on the Casimir of the adjoint representation, $c_{2}(A)$, originate from the s-channel diagram, and $s, t, u$ are the usual Mandelstam variables. For the case of real scalar fields, Eq. (5) must be multiplied by an additional factor of $1 / 2$. Many detailed phenomenological studies for octets, particularly electroweak doublets, exist in the literature [9-15], and we have checked that the corresponding results agree with ours. In particular, Eq. (5) is half as large as the corresponding cross section for squark pair production from gluon fusion for which there are two complex scalar doublets [16].

In Fig. 2, we present the leading-order $p p \rightarrow S S$ cross section for all representations satisfying Eq. (4) at the LHC with $\sqrt{s}=13 \mathrm{TeV}$ using the PDF4LHC15_nlo_mc parton distribution function set [17] with the factorization scale $\mu_{\mathrm{F}}=2 m_{S}$, accessed via the ManeParse package [18] and LHAPDF6 [19]. Calculations of these cross sections

\footnotetext{
${ }^{1}$ Similar considerations have been used for fermions in higher color representations $[7,8]$.
}

beyond leading order exist for the triplets (squarks) [20], where NLO enhancements over the LO are found to be described approximately by modest $K$ factors near 1.3 at $\sqrt{s}=14 \mathrm{TeV}$ with weak dependence on the squark mass. Next-to-leading order calculations for scalar octets (sgluons) exist in the literature [21] as well, and a $K$ factor in the range (1.5-1.8) is found at $\sqrt{s}=14 \mathrm{TeV}$, with the variation stemming from the sgluon mass. Leading-order

TABLE I. Dynkin indices of all representations of colored scalars allowed by asymptotic freedom.

\begin{tabular}{ccc}
\hline \hline Label & Representation & Dynkin index $t_{2}$ \\
\hline$(0,0)$ & $\mathbf{1}$ & 0 \\
$(1,0)$ & $\mathbf{3}$ & $1 / 2$ \\
$(2,0)$ & $\mathbf{6}$ & $5 / 2$ \\
$(1,1)$ & $\mathbf{8}$ & 3 \\
$(3,0)$ & $\mathbf{1 0}$ & $15 / 2$ \\
$(2,1)$ & $\mathbf{1 5}$ & 10 \\
$(4,0)$ & $\mathbf{1 5}^{\prime}$ & $35 / 2$ \\
\hline \hline
\end{tabular}



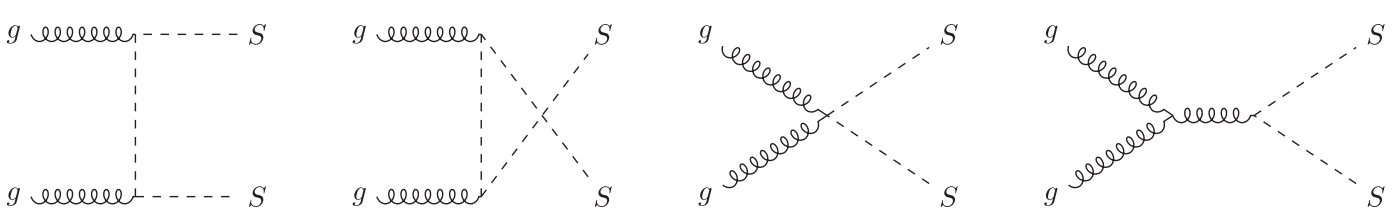

FIG. 1. Leading-order diagrams for scalar pair production from gluon fusion.

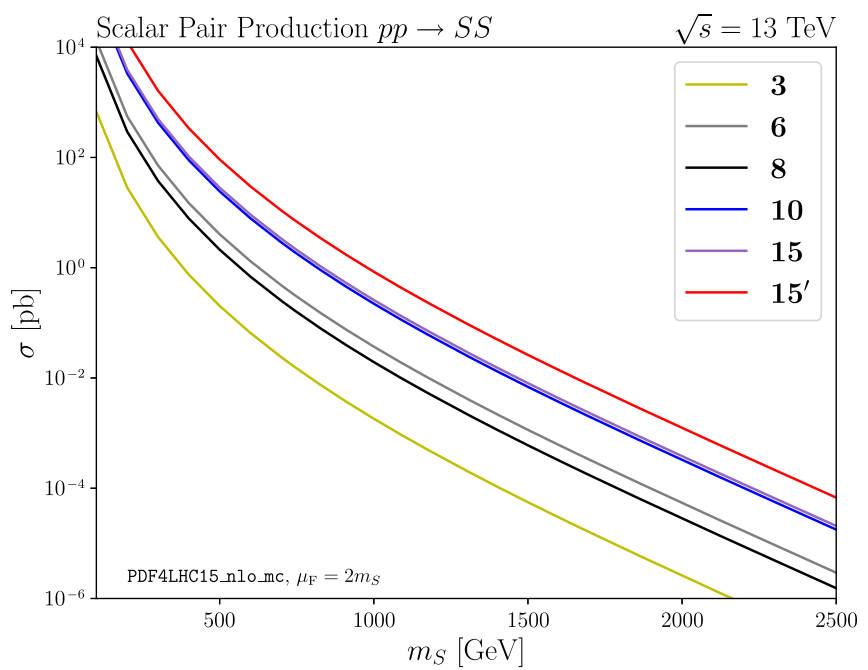

FIG. 2. Leading-order scalar pair production cross section $p p \rightarrow S S$ at the LHC with $\sqrt{s}=13 \mathrm{TeV}$ for complex scalars in $\mathrm{SU}(3)$ representations $3, \mathbf{6}, \mathbf{1 0}, \mathbf{1 5}, \mathbf{1 5}^{\prime}$ and real scalars in the adjoint representation $\mathbf{8}$.

color sextet (diquark) production at the LHC has been studied previously in [22].

\section{THE SCALAR POTENTIAL}

The renormalizable interactions of colored scalars are governed by the most-general scalar potential up to dimension four. For electroweak singlets, the terms,

$$
V_{R}=\kappa_{R} H^{\dagger} H S^{\dagger} S+\mu_{R}\left(S^{\dagger} S\right)^{2},
$$

occur for all SU(3) representations. There are also further representation-dependent terms, which will be detailed below for specific cases.

Triple-scalar couplings are allowed when $R \otimes$ $R \otimes R \supset \mathbf{1}$, and this is possible for all the representations we consider, as can be seen from Table III in the Appendix. However, some of these couplings are completely antisymmetric under scalar exchanges and therefore, vanish when the scalars carry no other quantum numbers beyond color. Completely symmetric triple-scalar couplings occur except for the $\mathbf{3}$ and $\mathbf{1 0}$ as shown below. We denote symmetric (antisymmetric) triple couplings by $\lambda_{R}\left(\tilde{\lambda}_{R}\right)$. The triple-scalar couplings allowed under these conditions have dimensions of mass, but they are not related to the usual Higgs vacuum expectation value. These presumably originate from the mechanism that gives mass to the scalars.

Model-dependent quartic-scalar couplings occur if $R \otimes R \otimes R \otimes R \supset 1$, and this occurs for $R=\mathbf{8}, \mathbf{1 0}$. In addition, for $R=\mathbf{1 0}$, there is a quartic coupling involving three scalar particles and one antiparticle.

In the following, fundamental indices of the color group are denoted by small latin letters $i, j, k, \ldots$, while adjoint indices are denoted by capital latin letters $A, B, C, \ldots$. The potentials for $R=\mathbf{3}, \mathbf{6}$, motivated by minimal flavor violation, have been discussed in [23] albeit with nonzero $U(1)_{Y}$ charge.

\section{A. Triplets}

It is possible to construct a singlet from three triplets but not from four so the most general potential is

$$
\begin{aligned}
V_{\mathbf{3}}= & m_{S}^{2} S_{i}^{\dagger} S^{i}+\kappa_{\mathbf{3}} H^{\dagger} H S_{i}^{\dagger} S^{i}+\mu_{\mathbf{3}}\left(S_{i}^{\dagger} S^{i}\right)^{2} \\
& +\left(\tilde{\lambda}_{\mathbf{3}} \varepsilon_{i j k} S^{i} S^{j} S^{k}+\text { H.c. }\right) .
\end{aligned}
$$

Note that the $\tilde{\lambda}_{3}$ term is completely antisymmetric and therefore vanishes for identical scalars.

\section{B. Sextets}

For scalar sextets it is once again possible to construct a singlet with three fields but not four, so the most general potential is given by

$$
\begin{aligned}
V_{\mathbf{6}}= & m_{S}^{2} S_{i j}^{\dagger} S^{i j}+\kappa_{\mathbf{6}} H^{\dagger} H S_{i j}^{\dagger} S^{i j}+\mu_{\mathbf{6} a}\left(S_{i j}^{\dagger} S^{i j}\right)^{2} \\
& +\mu_{\mathbf{6} b} S_{i k}^{\dagger} S_{j \ell}^{\dagger} S^{i j} S^{k \ell}+\left(\lambda_{\mathbf{6}} \varepsilon_{i k m} \varepsilon_{j \ell n} S^{i j} S^{k \ell} S^{m n}+\text { H.c. }\right) .
\end{aligned}
$$

In this case, the triple-scalar coupling is symmetric under the exchange of any two sextets.

\section{Octets}

The octet is a real representation of $\mathrm{SU}(3)$ and, in the absence of additional quantum numbers, we will use a real scalar field. As the octet plays a special role as the adjoint representation, we write the potential for this case using adjoint indices, ${ }^{2}$

\footnotetext{
${ }^{2}$ A real scalar in the model of [9] is $S_{R}$ and the triple-scalar coupling would correspond to $\lambda_{8}=v\left(\lambda_{4}+\lambda_{5}\right) / 12$ in that model.
} 


$$
V_{\mathbf{8}}=m_{S}^{2} S^{A} S^{A}+\kappa_{\mathbf{8}} H^{\dagger} H S^{A} S^{A}+\mu_{\mathbf{8}}\left(S^{A} S^{A}\right)^{2}+\lambda_{\mathbf{8}} d_{A B C} S^{A} S^{B} S^{C}
$$

Another quartic term found in the literature, $\sim \operatorname{Tr}\left(T^{A} T^{B} T^{C} T^{D}\right) S^{A} S^{B} S^{C} S^{D}$ [10,24], reduces to $\mu_{8}$ when the scalars do not carry other quantum numbers.

\section{Decuplets}

For scalar decuplets, the most general potential is given by

$$
\begin{aligned}
V_{\mathbf{1 0}}= & m_{S}^{2} S_{i j k}^{\dagger} S^{i j k}+\kappa_{\mathbf{1 0}} H^{\dagger} H S_{i j k}^{\dagger} S^{i j k}+\mu_{\mathbf{1 0} a}\left(S_{i j k}^{\dagger} S^{i j k}\right)^{2}+\mu_{\mathbf{1 0} b} S_{i j k}^{\dagger} S_{\ell m n}^{\dagger} S^{i j m} S^{\ell n k}+\left(\tilde{\lambda}_{\mathbf{1 0}} \varepsilon_{i \ell o} \varepsilon_{j m p} \varepsilon_{k n q} S^{i j k} S^{\ell m n} S^{o p q}\right. \\
& \left.+\omega_{\mathbf{1 0}} \varepsilon_{i \ell o} \varepsilon_{j m r} \varepsilon_{k p s} \varepsilon_{n q t} S^{i j k} S^{\ell m n} S^{o p q} S^{r s t}+\rho_{\mathbf{1 0}} S_{k n q}^{\dagger} \varepsilon_{i \ell o} \varepsilon_{j m p} S^{i j k} S^{\ell m n} S^{o p q}+\text { H.c. }\right)
\end{aligned}
$$

This color structure allows an antisymmetric triple-scalar vertex, a second quartic vertex with two particles and two antiparticles, a quartic vertex with four particles, and a quartic vertex with three particles and one antiparticle.

\section{E. (2, 1)-Quindecuplets}

For scalars transforming in the $\mathbf{1 5}$, we find the potential,

$$
\begin{aligned}
V_{\mathbf{1 5}}= & m_{S}^{2} S_{i j}^{\dagger k} S_{k}^{i j}+\kappa_{\mathbf{1 5}} H^{\dagger} H S_{i j}^{\dagger k} S_{k}^{i j}+\mu_{15 a}\left(S_{i j}^{\dagger k} S_{k}^{i j}\right)^{2}+\mu_{\mathbf{1 5} b}\left(S_{i j}^{\dagger k} S_{m n}^{\dagger \ell} S_{k}^{i m} S_{\ell}^{j n}\right)+\mu_{\mathbf{1 5}}\left(S_{i j}^{\dagger k} S_{m n}^{\dagger \ell} S_{\ell}^{i m} S_{k}^{j n}\right) \\
& +\left(\lambda_{\mathbf{1 5}} \varepsilon_{\ell m n} S_{j}^{i \ell} S_{k}^{j m} S_{i}^{k n}+\tilde{\lambda}_{\mathbf{1 5}} \varepsilon_{i j k} \varepsilon_{\ell m n} \varepsilon^{o p q} S_{o}^{i \ell} S_{p}^{j m} S_{q}^{k n}+\text { H.c. }\right) .
\end{aligned}
$$

The $(2,1)$ representation allows a symmetric triple-scalar vertex, the term with coupling $\lambda_{15}$; as well as an antisymmetric one with coupling $\tilde{\lambda}_{15}$. It does not allow quartic terms with three particles and one antiparticle nor ones with four particles.

\section{F. (4, 0)-Quindecuplets}

For scalars transforming in the $\mathbf{1 5}^{\prime}$, we find the potential,

$$
\begin{aligned}
V_{1 \mathbf{5}^{\prime}}= & m_{S}^{2} S_{i j k \ell}^{\dagger} S^{i j k \ell}+\kappa_{\mathbf{1 5}^{\prime}} H^{\dagger} H S_{i j k \ell}^{\dagger} S^{i j k \ell}+\mu_{1 \mathbf{5}^{\prime} a}\left(S_{i j k \ell}^{\dagger} S^{i j k \ell}\right)^{2}+\mu_{\mathbf{1 5}}\left(S_{i j k \ell}^{\dagger} S_{m n o p}^{\dagger} S^{i j m n} S^{k \ell o p}\right) \\
& +\mu_{1 \mathbf{5}^{\prime} c}\left(S_{i j k \ell}^{\dagger} S_{m n o p}^{\dagger} S^{i j k m} S^{\ell n o p}\right)+\left(\lambda_{\mathbf{1 5}^{\prime}} \varepsilon_{i \ell o} \varepsilon_{j m p} \varepsilon_{k n q} \varepsilon_{r s t} S^{i j k r} S^{\ell m n s} S^{o p q t}+\text { H.c. }\right) .
\end{aligned}
$$

The $(4,0)$ representation permits a symmetric triple-scalar coupling but no quartic couplings.

We want to close this section by elaborating upon possible Yukawa couplings of the colored scalars. Since we do not consider any Standard-Model extensions beyond the colored scalars, we only have the SM fermion content at our disposal, meaning that all fermions must either be color singlets or triplets. In effect, only scalar singlets, triplets, sextets, and octets can have Yukawa couplings. Moreover, if the scalars are $\mathrm{SU}(2)_{L}$ singlets and do not carry electric charge, the only possible Yukawa coupling is to right-handed neutrinos. Insisting on $\mathrm{SU}(2)_{L}$ singlets but allowing for $Y=Q \neq 0$, the only possibilities with colored scalars are for triplets and sextets, such as [25]

$$
\begin{gathered}
V_{Y, \mathbf{3}} \supset g_{\mathrm{Y}, \mathbf{3}}^{e 1} S^{i} \bar{d}_{i R} e_{R}^{c}+g_{\mathrm{Y}, \mathbf{3}}^{e 2} S^{i} \bar{Q}_{i L} L_{L}^{c}+g_{\mathrm{Y}, \mathbf{3}}^{e 3} S^{i} \bar{u}_{i R} e_{R}^{c}+g_{\mathrm{Y}, \mathbf{3}}^{q 1} \varepsilon_{i j k} S^{i} \bar{Q}_{L}^{c} Q_{L} \\
+g_{\mathrm{Y}, \mathbf{3}}^{q 2} \varepsilon_{i j k} S^{i} \bar{u}_{R}^{c} d_{R}+g_{\mathrm{Y}, \mathbf{3}}^{q 3} \varepsilon_{i j k} S^{i} \bar{u}_{R}^{c} u_{R}+g_{\mathrm{Y}, \mathbf{3}}^{q 4} \varepsilon_{i j k} S^{i} \bar{d}_{R}^{c} d_{R}+\text { H.c. } \\
V_{Y, \mathbf{6}} \supset g_{\mathrm{Y}, \mathbf{6}}^{q 1} S^{i j} \bar{Q}_{i L} Q_{j L}^{c}+g_{\mathrm{Y}, \mathbf{6}}^{q 2} S^{i j} \bar{d}_{i R} u_{j R}^{c}+g_{\mathrm{Y}, \mathbf{6}}^{q 3} S^{i j} \bar{u}_{i R} u_{j R}^{c}+g_{\mathrm{Y}, \mathbf{6}}^{q 4} S^{i j} \bar{d}_{i R} d_{j R}^{c}+\text { H.c., }
\end{gathered}
$$

where we have omitted flavor indices. All of these couplings require the scalars to carry electric charge, so are not of interest here.

\section{SCALAR DECAY MODES}

We now examine possible decay modes at tree and one-loop level, and show that only the octet can decay. To conserve color, any scalar decay must include quarks, gluons, or more scalars. 

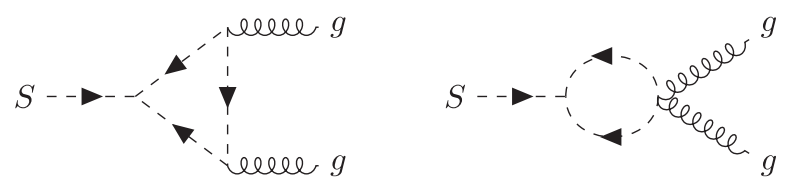

FIG. 3. Lowest-order loop-induced diagrams for $S \rightarrow g g$.

\section{A. Tree-level decays}

Decays into standard-model fermion pairs, $S \rightarrow f \bar{f}$, could proceed at tree level through Yukawa interactions. As the fermions in the SM transform under the color group as singlets or triplets, the $f \bar{f}$ state can only be an $\mathrm{SU}(3) \mathbf{1}, \mathbf{3}$, 6, or 8. Requiring that the new scalars be electroweak singlets forbids any couplings of the form listed in Eq. (14) so decays to fermion pairs are not possible. If instead, we allow scalars with non-zero hypercharge, these would decay into two or more jets for $\mathbf{3}, \mathbf{6}$, or $\mathbf{8}$ and small Yukawa couplings would be required to ensure longevity.

Since we consider only one (degenerate) multiplet at a time, the scalars cannot decay into each other either.

\section{B. One-loop decays}

The decay $S \rightarrow g g$ is in principle allowed as a loopinduced process. Without Yukawa couplings, however, it can only proceed via intermediate colored scalars as shown in Fig. 3. This process then depends on the existence of appropriate triple-scalar couplings and can proceed only for scalars in the octet, $\mathbf{8}$, as we will show below.

Color conservation indicates that only those representations that appear in the Clebsch-Gordan decomposition of the direct product of two adjoint representations can decay into two gluons,

$$
\mathbf{8} \otimes \mathbf{8}=\mathbf{1} \oplus_{2} \mathbf{8} \oplus \mathbf{1 0} \oplus \overline{\mathbf{1 0}} \oplus \mathbf{2 7}
$$

Of these, we only need to consider the adjoint $\mathbf{8}$ and (anti) decuplet $10(\overline{\mathbf{1 0}})$ representations as per Eq. (4). It is straightforward to confirm that these are the only representations to consider even if a few additional gluons are added in the final state.

We begin by examining the case of the $\mathbf{1 0}$ (or $\overline{\mathbf{1 0}}$ ). The color flow (indicated by the arrows) shows that for the lefthand diagram in Fig. 3 to be nonvanishing, either an $S S g$ or an $S^{\dagger} S^{\dagger} g$ vertex is required, while for the seagull diagram in Fig. 3 an $S S g g$ vertex is needed. However, gluons only appear in the quadratic part of the Lagrangian which couples a $\mathbf{1 0}$ with a $\overline{\mathbf{1 0}}$ due to the constraint of the Lagrangian being a color singlet. Hence, neither of the one-loop diagrams appears for scalars transforming as (anti)decuplets. This argument indicates that the diagrams in Fig. 3 only occur for real representations. We note that the decuplet also cannot decay into two gluons at the twoloop level when it carries no quantum numbers other than color. This is true, because the required triple-scalar vertex,
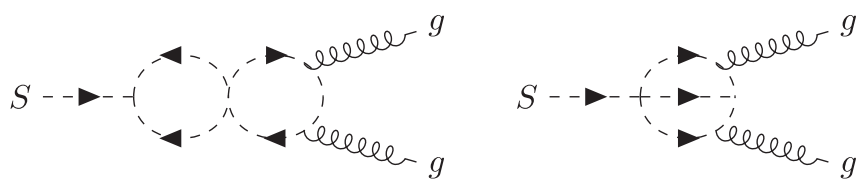

FIG. 4. Generic two-loop diagrams for the $S_{\mathbf{1 0}} \rightarrow g g$ decay combining vertices with $\kappa_{\mathbf{1 0}}$ and $\mu_{\mathbf{1 0}}^{\prime}$. Arrows indicate particles/ antiparticles (or color/anticolor representations). The gluons can be emitted from any of the scalar lines as shown or both at a time from a seagull vertex.

cf. Fig. 4 (the gluons can be attached to any scalar line), is antisymmetric and therefore, vanishes for identical scalars.

Following the above reasoning, the only possibility left for $S \rightarrow g g$ to occur is for color octets, where the answer at one-loop is already known. In adjoint indices, it is given by $[11,14]$

$$
\mathcal{L}_{S g g}=\frac{9 \alpha_{\mathrm{s}}}{8 \pi} \frac{\lambda_{\mathbf{8}}}{m_{S}^{2}} I_{S}(1) d_{A B C} G_{\mu \nu}^{A} G^{B \mu \nu} S^{C}
$$

where the loop factor is $I_{s}(1)=\left(\frac{\pi^{2}}{9}-1\right)$ for the case where all intervening scalars are degenerate in mass, cf. Appendix B. The decay width is then

$$
\Gamma(S \rightarrow g g)=\frac{9}{8 \pi}\left(\frac{\alpha_{\mathrm{s}}}{8 \pi}\right)^{2} \frac{\lambda_{\mathbf{8}}^{2}}{m_{S}} 30 I_{s}(1)^{2},
$$

with 30 being a color factor.

\section{Mixed decays to quarks and gluons}

Color conservation implies that the $S_{15}$ can decay into states like $q g$ or $q g g$ and the $S_{\mathbf{1 5}^{\prime}}$ into $q g g$. To proceed at dimension six (eight) these processes would require operators of the form $(\ell, q$ denote a generic lepton and quark, respectively),

$$
\mathcal{O} \sim \bar{\ell} \sigma_{\mu \nu} q G^{\mu \nu} S_{15}, \quad \mathcal{O} \sim \bar{\ell} q G^{\mu \nu} G_{\mu \nu} S_{15^{\prime}}
$$

where the gluons have to appear explicitly to satisfy the color requirement. The necessary fermion bilinears, however, cannot couple to scalars with zero hypercharge as we saw in our discussion of Yukawa couplings.

\section{CONSTRAINTS FROM $H \rightarrow g g$}

For all representations we have discussed here, there is an additional new contribution to the Higgs decay $H \rightarrow g g$ and correspondingly, to the Higgs boson production cross section from gluon fusion, proceeding through the diagrams in Fig. 5 [24,26]. This contribution will be proportional to the $H S S^{\dagger}$ coupling, which is contained in the term in the scalar potential proportional to $\kappa_{R}$ in Eq. (6). 

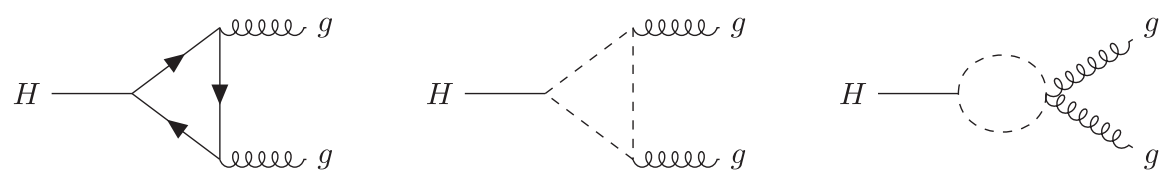

FIG. 5. Lowest-order loop-induced diagrams for the decay $H \rightarrow g g$, including the standard-model contribution mediated by heavy quarks (left) and new contributions mediated by colored scalars (middle and right).

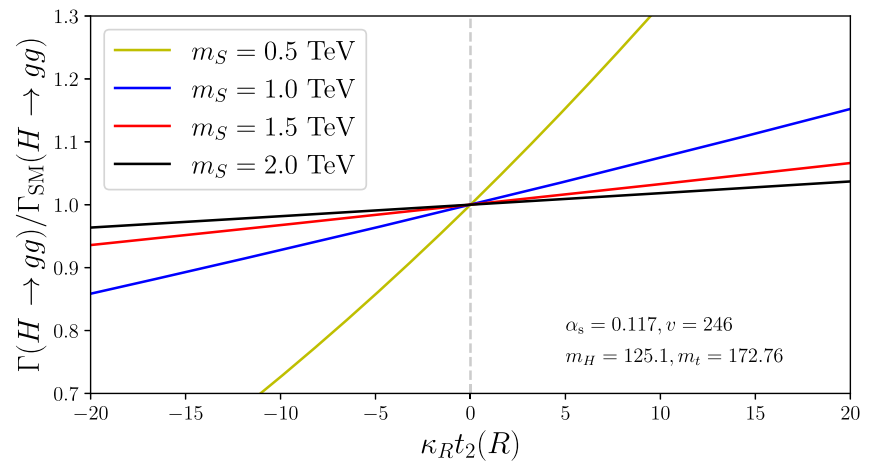

FIG. 6. Corrections to the decay $H \rightarrow g g$ for different scalar masses and representations.

After adding the SM top-quark loop contribution, we find $^{3}$

$$
\begin{aligned}
\Gamma(H \rightarrow g g)= & \frac{m_{H}^{3}}{8 \pi v^{2}}\left(\frac{\alpha_{\mathrm{s}}}{\pi}\right)^{2} \mid I_{q}\left(\frac{m_{t}^{2}}{m_{H}^{2}}\right) \\
& +\left.\kappa_{R} \frac{v^{2}}{4 m_{H}^{2}} t_{2}(R) I_{s}\left(\frac{m_{S}^{2}}{m_{H}^{2}}\right)\right|^{2},
\end{aligned}
$$

with the known loop functions listed in Appendix B. The corresponding corrections to the partial decay width of the Higgs $\Gamma(H \rightarrow g g)$ are illustrated in Fig. 6. From the figure, we see that for $\left|\kappa_{R}\right| \lesssim 1$, and for all the representations satisfying Eq. (4) scalars with masses $m_{S} \gtrsim 1 \mathrm{TeV}$ have modest effects. The parameter space for lighter scalars, on the other hand, can be significantly constrained by Higgs decays.

\section{EXOTIC SHOWERS AND HADRONS}

As shown above, electroweak singlet scalars in color representations $R=\mathbf{3}, \mathbf{6}, \mathbf{1 0}, \mathbf{1 5}, \mathbf{1 5}^{\prime}$ cannot decay and are therefore long-lived. The radiation of additional gluons in form of bremsstrahlung is, however, possible for all the scalars we have considered here. After production, these scalars will therefore lose energy via a QCD showering process and, once a scale of the order of the hadronization scale $\Lambda_{\mathrm{QCD}} \approx 1 \mathrm{GeV}$ is reached, hadronize by picking up quarks and gluons to form exotic color singlets. This situation is comparable to the one for so-called $R$ hadrons

\footnotetext{
${ }^{3}$ In this notation, for example, the complex $S^{ \pm}$scalar field in [9] would contribute with $\kappa_{R}=\frac{\lambda_{1}}{2}$ and $t_{2}(\mathbf{8})=3$.
}

[4,27], where exotic hadrons are formed from long-lived supersymmetric particles, such as the stop or the gluino. Irrespective of their origin, we will call such exotic hadrons $R$ hadrons in the following.

The simplest $R$ hadrons that can be formed from the long-lived scalars are collected in Table II with the corresponding Clebsch-Gordan decompositions shown in Table IV. Except for the decuplet, all scalars would form fractionally charged hadrons. In addition, color singlets containing the $(4,0)$-quindecuplet can only be formed with a minimum of three additional partons.

Another intriguing possibility is the formation of quarkoniumlike bound states following the production of scalar antiscalar pairs. This was considered in [28] for color octets, and we will not pursue it further.

\section{A. Phenomenological modeling}

Multipurpose event generators such as SHERPA [29], HERWIG [30], and PYTHIA [31] provide general tools for phenomenological studies of Standard Model phenomena as well as extensions to it. It would therefore be beneficial to implement our models considered above in such a framework to give access to a detailed modeling of not only the hard production process [as described by the leading-order cross section in Eq. (5)] but also the modeling of logarithmically enhanced QCD bremsstrahlung and nonperturbative effects, such as hadron fragmentation. In addition, Monte Carlo event generators provide the input for dedicated detector simulation programs, such as GEANT4 [32] or DELPHES [33,34], giving access to realistic particle-level analysis environments.

Monte Carlo event generators, however, routinely use the Les Houches standard to store color information [35], which only allows for two color tags per particle. (Strictly

TABLE II. List of possible $R$ hadrons for each long-lived representation. Here, $u$ and $d$ denote generic up- and down-type quarks, respectively.

\begin{tabular}{ll}
\hline \hline Representation & \multicolumn{1}{c}{$R$ hadrons } \\
\hline $\mathbf{3}$ & $R_{S \bar{u}}^{-2 / 3}, R_{S \bar{d}}^{+1 / 3}, R_{S u u}^{+4 / 3}, R_{S d d}^{-2 / 3}$ \\
$\mathbf{6}$ & $R_{S \bar{u} \bar{u}}^{-4 / 3}, R_{S \bar{d} \bar{d}}^{+2 / 3}, R_{S u g}^{+2 / 3}, R_{S d g}^{-1 / 3}$ \\
$\mathbf{1 0}$ & $R_{S g g}^{0}$ \\
$\mathbf{1 5}$ & $R_{S \bar{u} g}^{-2 / 3}, R_{S \bar{d} g}^{+1 / 3}$ \\
$\mathbf{1 5}^{\prime}$ & $R_{S \bar{u} g g}^{-2 / 3}, R_{S \bar{d} g g}^{+1 / 3}$ \\
\hline \hline
\end{tabular}


speaking, it only allows for a color-anticolor combination, but this can be tweaked to enable color-color or anticoloranticolor combinations, cf., e.g., [36].) Implementations of models with colored particles in representations other than the (anti)triplet or octet have therefore been limited mainly to sextets. An explicit "color flow" representation of the Standard Model vertices has been presented in [37] in the context of the O'Mega [38] matrix element generator in WHIZARD $[39,40]$. Sextet production including subsequent parton showering has been studied in [22], while a general shower framework for heavy colored particles has been developed in [41] and extended to showers off stops $\tilde{t}$ in [42]. As far as the nonperturbative modeling is concerned, both the HERWIG and PYTHIA event generation frameworks provide dedicated modules for the hadronization of longlived colored particles, in the form of $R$ hadrons, cf. [36,43] for a description of the model implemented in PYTHIA8. A comparison of PYTHIA's string and HERWIG's cluster fragmentation models for the case of $R$ hadrons was considered in [44].

A Monte Carlo implementation of the decuplet and quindecuplet models considered in this work necessitates the extension of the format in which color information is stored and interpreted in event generators. As the bare minimum, a third color tag has to be introduced and leadingorder matrix elements projected onto a "leading color," i.e., planar, decomposition in terms of these. It must be emphasized that while this may enable the parton showering of such colored particles, it does not automatically include nonperturbative modeling of showered events. To this end, hadronization models require a more fundamental extension to utilize the additional color representations in the formation of color-singlet hadrons. Such an implementation is therefore beyond the scope of this work. We will instead obtain a first estimate of LHC bounds in the next section.

\section{B. A first estimate of LHC limits}

To estimate the limits that could be placed at LHC, we can readily adapt some of the existing results for $R$ hadrons found in the literature, cf., e.g., [45-47].

The ATLAS experiment, using $36.1 \mathrm{fb}^{-1}$ of data at $\sqrt{s}=13 \mathrm{TeV}$, rules out at the $95 \%$ C.L. long-lived sbottom and stop $R$ hadrons with masses below $1250 \mathrm{GeV}$ and $1340 \mathrm{GeV}$, respectively. The production cross section for sbottom or stop pairs from gluon fusion ${ }^{4}$ is twice as large as the one shown in yellow for color triplets in Fig. 2. Differences in the lifetime are not important for these constraints, as the scalars considered here are stable, and those in [46] are assumed to live long enough to reach the hadronic calorimeter (decay lengths of a few meters).

\footnotetext{
${ }^{4}$ The leading-order gluon-fusion partonic cross section for complex triplet scalars is the same as for squarks, but the one for octets is smaller than the one for gluinos, see, for example, $[16,20]$.
}

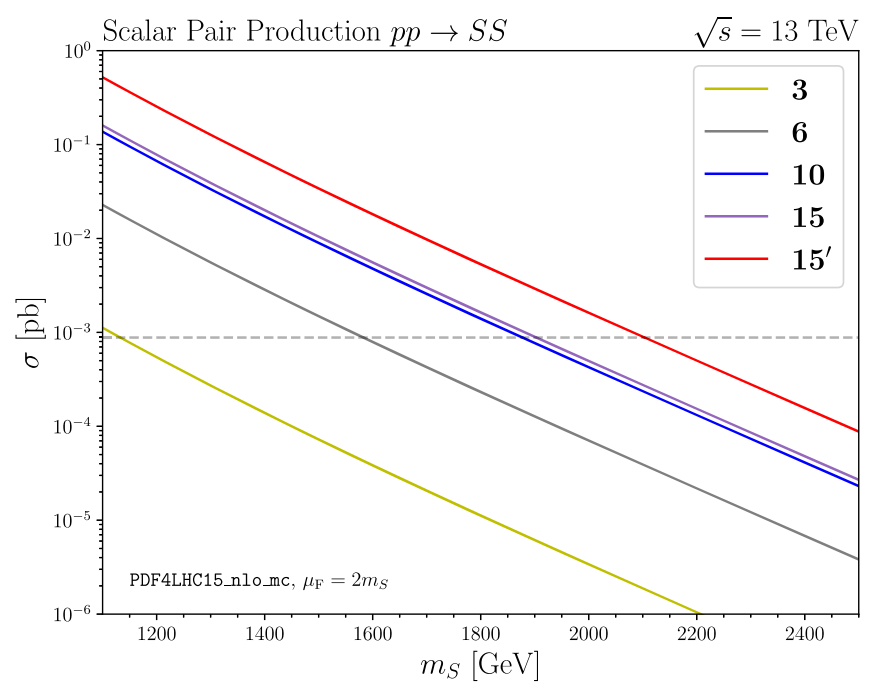

FIG. 7. Pair-production cross sections for all long-lived scalars including a $K$ factor of 1.3 , with the dashed line marking a firstestimate LHC bound.

For comparison, searches (with significantly lower integrated luminosity) assuming the particles to be stable find an upper bound for stop $R$ hadrons near $1 \mathrm{TeV}$, cf. the respective CMS [48] and ATLAS [49] publications. The main difference would be in the constituents of the color-singlet exotic hadron. Whereas for sbottom and stop we could have neutral objects, for the color triplet considered here the exotic hadron would have a fractional electric charge, cf. Table II. CMS searches looking for long-lived fractionally charged objects find similar constraints as the ones above, cf. $[48,50]$. We thus expect bounds for exotic hadrons from color triplets to be around a $\mathrm{TeV}$, slightly weaker than those for squarks, with stronger limits for the higher representations.

To obtain a first estimate for the scalar-mass constraints, we compare our scalar pair-production cross sections to the average cross section corresponding to the ATLAS sbottom and stop $R$-hadron mass limits. In addition, we include a $K$ factor of 1.3 for all representations. The results are shown in Fig. 7, where we have superimposed the first-estimate exclusion cross section as a horizontal line. From this figure, the expected constraints based only on a scaling of the cross section can be read off to range from $m_{S}=1.1 \mathrm{TeV}$ for color triplets to $m_{S} \approx 2.1 \mathrm{TeV}$ for scalars in the $\mathbf{1 5}^{\prime}$.

As LHC limits for long-lived charged particles rely on measurements of ionization and as such do not directly apply to our neutral decuplet $R_{S g g}^{0}$, some remarks are in order:

(i) First, the scalar decuplet can also hadronize with three quarks leading to a charged $R_{\text {Sǖ } \bar{d}}^{+}$, for example. The hadronization ratios into the different possible $R$ hadrons are not known but are expected to be comparable. In addition, collisions of an $R$ hadron with the detector material can change the electric charge through strong interaction reactions such as $R_{S g g}^{0}+p \rightarrow R_{S \bar{u} \bar{u} \bar{d}}^{+}+$pions. 
(ii) The neutral $R$ hadrons would also leave a hadronic shower in the hadronic calorimeters providing another handle for their detection.

It is interesting to note that one can also obtain an upper limit on the mass of neutral $R$ hadrons by requiring their annihilation cross section be large enough to avoid overproduction of relic dark matter density [51,52]. We can estimate the annihilation cross section in a spectator model by adjusting the kinematic and color-average factors in Eq. (5) to find

$\left\langle\sigma\left(R_{S g g}^{0} R_{S g g}^{0} \rightarrow g g\right) v\right\rangle \approx \frac{\pi \alpha_{S}^{2} c_{2}(R)}{8(\operatorname{dim} R) m_{S}^{2}}\left(4 c_{2}(R)-c_{2}(A)\right)$.

This result, in combination with $\langle\sigma v\rangle \gtrsim 4 \times 10^{-9} \mathrm{GeV}^{-2}$ [51], suggests that $m_{R_{S g g}^{0}} \lesssim 3.2 \mathrm{TeV}$. There is no lower bound in this case since there could be many other contributions to the relic density.

\section{SUMMARY AND CONCLUSIONS}

In this manuscript, we have discussed scalar extensions to the Standard Model, transforming nontrivially under the color group and as singlets under the electroweak gauge group. Based on the requirement to leave asymptotic freedom intact, the maximal dimension of the color representation is 15 . Requiring the new scalars to have zero hypercharge, the new particles are stable, with only octets decaying to gluons at the one-loop level.

We have discussed how long-lived colored scalars will appear as exotic hadrons in detector signals and obtained a first estimate of mass bounds at the LHC. More detailed analyses can be obtained by implementing models of higher color representations in multipurpose event generators, and we have outlined such implementations, including a discussion of current technical restrictions. The implementation in multipurpose event generators is beyond the scope of the current work but will be important to obtain mass constraints from collider experiments, as it facilitates the modeling of QCD as well as EW/QED bremsstrahlung, hadronization as well as other nonperturbative corrections, and subsequent detector simulation.

\section{ACKNOWLEDGMENTS}

We would like to thank Ulrik Egede, Xiao-Gang He, Peter Skands, and Ray Volkas for helpful discussions. C. T. P. is supported by the Monash Graduate Scholarship, the Monash International Postgraduate Research Scholarship, and the J. L. William Scholarship.

\section{APPENDIX A: SOME SU(3) RELATIONS AND NOTATION}

For a general representation $R$, the Dynkin index is given by the trace of the generators $T_{R}$ in the representation,

$$
\operatorname{Tr}\left(T_{R}^{A} T_{R}^{B}\right)=t_{2}(R) \delta^{A B},
$$

and is related to the eigenvalue $c_{2}(R)$ of the quadratic Casimir operator,

$$
c_{2}(R)=\delta^{A B} T_{R}^{A} T_{R}^{B},
$$

in the representation $R$ by

$$
t_{2}(R)=\frac{\operatorname{dim} R}{\operatorname{dim} \mathfrak{g} \mathfrak{u}(3)} c_{2}(R),
$$

where $\operatorname{dim} R$ and $\operatorname{dim} \mathfrak{g} \mathfrak{u}(3)=8$ denote the dimensions of the representation and the Lie algebra $\mathfrak{g} \mathfrak{t}(3)$ of the gauge group $\mathrm{SU}(3)$, respectively. Labeling irreducible representations $R(p, q)$ of $\mathfrak{g} \mathfrak{t}(3)$ by the Dynkin label $(p, q)$, the eigenvalue of the quadratic Casimir is

$c_{2}(R(p, q)) \equiv c_{2}(p, q)=\frac{p^{2}+q^{2}+3 p+3 q+p q}{3}$.

Moreover, the dimension of the representation $R(p, q)$ can be calculated by

$$
\operatorname{dim} R(p, q)=\frac{(p+1)(q+1)(p+q+2)}{2} .
$$

In the Standard Model, gluons transform with respect to the adjoint representation $\mathbf{8}$ and fermions with respect to the fundamental representation 3. Therefore, with $n_{\mathrm{F}}=6$, $N_{\mathrm{C}}=3$, and $T_{\mathrm{R}}=1 / 2$, in the Standard Model, we have

$$
t_{2}(V)=t_{2}(\mathbf{8})=3, \quad \text { and } \quad t_{2}(F)=t_{2}(\mathbf{3})=\frac{1}{2} .
$$

Some Clebsch-Gordan decompositions useful to determine the terms entering the scalar potential are tabulated below. All decompositions are calculated with LieART [53] and cross-checked with an independent implementation of the algorithm in [54].

\section{APPENDIX B: SCALAR LOOP FUNCTIONS}

In this appendix, we collect the well-known scalar oneloop functions appearing in the loop-induced diagrams $H \rightarrow g g$ and $S \rightarrow g g$.

$$
\begin{aligned}
& I_{q}(x)=2 x-x(1-4 x) f(x), \quad I_{s}(x)=-(1+2 x f(x)), \\
& f(x)= \begin{cases}\frac{1}{2}\left(\ln \left(\frac{1+\sqrt{1-4 x}}{1-\sqrt{1-4 x}}\right)-i \pi\right)^{2} & \text { for } x<\frac{1}{4} \\
-2\left(\arcsin \left(\frac{1}{2 \sqrt{x}}\right)\right)^{2} & \text { for } x>\frac{1}{4}\end{cases}
\end{aligned}
$$


TABLE III. Selected three- and four-particle Clebsch-Gordan decompositions.

\begin{tabular}{|c|c|}
\hline Direct Product & Decomposition \\
\hline $3 \otimes 3 \otimes 3$ & $\mathbf{1} \oplus_{2} \mathbf{8} \oplus \mathbf{1 0}$ \\
\hline $6 \otimes 6 \otimes 6$ & $\mathbf{1} \oplus_{2} \mathbf{8} \oplus \mathbf{1 0} \oplus \overline{\mathbf{1 0}} \oplus_{3} \mathbf{2 7} \oplus \mathbf{2 8} \oplus_{2} 35$ \\
\hline $8 \otimes 8 \otimes 8$ & $\oplus_{2} \mathbf{1} \oplus_{8} \mathbf{8} \oplus_{4} \mathbf{1 0} \oplus_{4} \overline{\mathbf{1 0}} \oplus_{6} \mathbf{2 7} \oplus_{2} \mathbf{3 5} \oplus_{2} \overline{\mathbf{3 5}} \oplus \mathbf{6 4}$ \\
\hline $10 \otimes 10 \otimes 10$ & $\mathbf{1} \oplus_{2} \mathbf{8} \oplus \mathbf{1 0} \oplus \overline{\mathbf{1 0}} \oplus_{3} \mathbf{2 7} \oplus \mathbf{2 8} \oplus_{2} \mathbf{3 5} \oplus_{2} \overline{\mathbf{3 5}} \oplus \cdots$ \\
\hline $15 \otimes 15 \otimes 15$ & $\oplus_{2} \mathbf{1} \oplus_{10} \mathbf{8} \oplus_{8} \mathbf{1 0} \oplus_{7} \overline{\mathbf{1 0}} \oplus_{15} \mathbf{2 7} \oplus_{4} \mathbf{2 8} \oplus \overline{\mathbf{2 8}} \oplus_{11} \mathbf{3 5} \oplus_{8} \overline{\mathbf{3 5}} \oplus \cdots$ \\
\hline $15^{\prime} \otimes 15^{\prime} \otimes 15^{\prime}$ & $\mathbf{1} \oplus_{2} \mathbf{8} \oplus \mathbf{1 0} \oplus \overline{\mathbf{1 0}} \oplus_{3} \mathbf{2 7} \oplus \mathbf{2 8} \oplus \overline{\mathbf{2 8}} \oplus_{2} \mathbf{3 5} \oplus_{2} \overline{\mathbf{3 5}} \oplus \cdots$ \\
\hline $8 \otimes 8 \otimes 8 \otimes 8$ & $\oplus_{8} \mathbf{1} \oplus_{32} \mathbf{8} \oplus_{20} \mathbf{1 0} \oplus_{20} \overline{\mathbf{1 0}} \oplus_{33} \mathbf{2 7} \oplus_{2} \mathbf{2 8} \oplus_{2} \overline{\mathbf{2 8}} \oplus_{15} \mathbf{3 5} \oplus_{15} \overline{\mathbf{3 5}} \oplus \cdots$ \\
\hline $10 \otimes 10 \otimes 10 \otimes 10$ & $\mathbf{1} \oplus_{8} \mathbf{8} \oplus_{10} \mathbf{1 0} \oplus_{6} \overline{\mathbf{1 0}} \oplus_{15} \mathbf{2 7} \oplus_{6} \mathbf{2 8} \oplus_{4} \overline{\mathbf{2 8}} \oplus \cdots$ \\
\hline $10 \otimes 10 \otimes 10 \otimes \overline{10}$ & $\mathbf{1} \oplus_{8} \mathbf{8} \oplus_{7} \mathbf{1 0} \oplus_{10} \overline{\mathbf{1 0}} \oplus_{18} \mathbf{2 7} \oplus_{10} \mathbf{2 8} \oplus_{3} \overline{\mathbf{2 8}} \oplus \cdots$ \\
\hline
\end{tabular}

TABLE IV. Relevant Clebsch-Gordan decompositions needed to form a color-singlet bound state with one colored scalar.

\begin{tabular}{|c|c|}
\hline Direct Product & Decomposition \\
\hline $3 \otimes \overline{\mathbf{3}}$ & $1 \oplus 8$ \\
\hline $8 \otimes 8$ & $\mathbf{1} \oplus_{2} \mathbf{8} \oplus \mathbf{1 0} \oplus \overline{\mathbf{1 0}} \oplus \mathbf{2 7}$ \\
\hline $3 \otimes 3 \otimes 3$ & $1 \oplus_{2} \mathbf{8} \oplus \mathbf{1 0}$ \\
\hline $3 \otimes 3 \otimes 8$ & $\mathbf{1} \oplus_{3} \mathbf{8} \oplus \mathbf{1 0} \oplus \overline{\mathbf{1 0}} \oplus \mathbf{2 7}$ \\
\hline $3 \otimes 6 \otimes 8$ & $\mathbf{1} \oplus_{3} \mathbf{8} \oplus_{2} \mathbf{1 0} \oplus \overline{\mathbf{1 0}} \oplus_{2} 27 \oplus \mathbf{3 5}$ \\
\hline$\overline{\mathbf{3}} \otimes \overline{\mathbf{3}} \otimes \mathbf{6}$ & $1 \oplus_{2} \mathbf{8} \oplus \mathbf{1 0} \oplus \mathbf{2 7}$ \\
\hline$\overline{3} \otimes 8 \otimes 15$ & $\mathbf{1} \oplus_{4} \mathbf{8} \oplus_{3} \mathbf{1 0} \oplus_{2} \overline{\mathbf{1 0}} \oplus_{4} \mathbf{2 7} \oplus \cdots$ \\
\hline $8 \otimes 8 \otimes 10$ & $\mathbf{1} \oplus_{4} \mathbf{8} \oplus_{4} \mathbf{1 0} \oplus_{2} \overline{\mathbf{1 0}} \oplus_{5} \mathbf{2 7} \oplus \cdots$ \\
\hline$\overline{3} \otimes 8 \otimes 8 \otimes 15^{\prime}$ & $\mathbf{1} \oplus_{6} \mathbf{8} \oplus_{8} \mathbf{1 0} \oplus_{3} \overline{\mathbf{1 0}} \oplus_{11} 27 \oplus \cdots$ \\
\hline
\end{tabular}

[1] O. Eberhardt, V. Miralles, and A. Pich, Constraints on coloured scalars from global fits, J. High Energy Phys. 10 (2021) 123.

[2] J. Alimena et al., Searching for long-lived particles beyond the Standard Model at the Large Hadron Collider, J. Phys. G 47, 090501 (2020).

[3] D. Curtin et al., Long-lived particles at the energy frontier: The MATHUSLA physics case, Rep. Prog. Phys. 82, 116201 (2019).

[4] G. R. Farrar and P. Fayet, Phenomenology of the production, decay, and detection of new hadronic states associated with supersymmetry, Phys. Lett. 76B, 575 (1978).

[5] J. Barnard, P. Cox, T. Gherghetta, and A. Spray, Long-lived, colour-triplet scalars from unnaturalness, J. High Energy Phys. 03 (2016) 003.

[6] A. de la Puente and A. Szynkman, Long-lived colored Scalars at the LHC, Eur. Phys. J. C 76, 124 (2016).

[7] W. J. Marciano, Exotic new quarks and dynamical symmetry breaking, Phys. Rev. D 21, 2425 (1980).
[8] R. Chivukula, M. Golden, and E. H. Simmons, Six jet signals of highly colored fermions, Phys. Lett. B 257, 403 (1991).

[9] A. V. Manohar and M. B. Wise, Flavor changing neutral currents, an extended scalar sector, and the Higgs production rate at the CERN LHC, Phys. Rev. D 74, 035009 (2006).

[10] N. V. Krasnikov, The phenomenology of scalar color octets, JETP Lett. 62, 7 (1995).

[11] M. I. Gresham and M. B. Wise, Color octet scalar production at the LHC, Phys. Rev. D 76, 075003 (2007).

[12] T. Han, I. Lewis, and Z. Liu, Colored resonant signals at the LHC: Largest rate and simplest topology, J. High Energy Phys. 12 (2010) 085.

[13] A. Hayreter and G. Valencia, LHC constraints on color octet scalars, Phys. Rev. D 96, 035004 (2017).

[14] A. Hayreter and G. Valencia, Color-octet scalar decays to a gluon and an electroweak gauge boson in the Manohar-Wise model, Phys. Rev. D 102, 115033 (2020). 
[15] V. Miralles and A. Pich, LHC bounds on colored scalars, Phys. Rev. D 100, 115042 (2019).

[16] S. Dawson, E. Eichten, and C. Quigg, Search for supersymmetric particles in hadron-hadron collisions, Phys. Rev. D 31, 1581 (1985).

[17] J. Butterworth et al., PDF4LHC recommendations for LHC Run II, J. Phys. G 43, 023001 (2016).

[18] D. B. Clark, E. Godat, and F. I. Olness, ManeParse: A Mathematica reader for parton distribution functions, Comput. Phys. Commun. 216, 126 (2017).

[19] A. Buckley, J. Ferrando, S. Lloyd, K. Nordström, B. Page, M. Rüfenacht et al., LHAPDF6: parton density access in the LHC precision era, Eur. Phys. J. C 75, 132 (2015).

[20] W. Beenakker, R. Hopker, M. Spira, and P. M. Zerwas, Squark and gluino production at hadron colliders, Nucl. Phys. B492, 51 (1997).

[21] D. Goncalves-Netto, D. Lopez-Val, K. Mawatari, T. Plehn, and I. Wigmore, Sgluon pair production to next-to-leading order, Phys. Rev. D 85, 114024 (2012).

[22] P. Richardson and D. Winn, Simulation of sextet diquark production, Eur. Phys. J. C 72, 1862 (2012).

[23] J. M. Arnold, M. Pospelov, M. Trott, and M. B. Wise, Scalar representations and minimal flavor violation, J. High Energy Phys. 01 (2010) 073.

[24] B. A. Dobrescu, G. D. Kribs, and A. Martin, Higgs Underproduction at the LHC, Phys. Rev. D 85, 074031 (2012).

[25] A. Davies and X.-G. He, Tree level scalar fermion interactions consistent with the symmetries of the standard model, Phys. Rev. D 43, 225 (1991).

[26] X.-G. He and G. Valencia, An extended scalar sector to address the tension between a fourth generation and Higgs searches at the LHC, Phys. Lett. B 707, 381 (2012).

[27] G. R. Farrar, R. Mackeprang, D. Milstead, and J. P. Roberts, Limit on the mass of a long-lived or stable gluino, J. High Energy Phys. 02 (2011) 018.

[28] A. Idilbi, C. Kim, and T. Mehen, Pair production of color-octet scalars at the LHC, Phys. Rev. D 82, 075017 (2010).

[29] SHERPA Collaboration, Event generation with SHERPA2.2, SciPost Phys. 7, 034 (2019).

[30] J. Bellm et al., HERwiG7.2 release note, Eur. Phys. J. C 80, 452 (2020).

[31] T. Sjöstrand, S. Ask, J. R. Christiansen, R. Corke, N. Desai, P. Ilten et al., An introduction to PYTHIA8.2, Comput. Phys. Commun. 191, 159 (2015).

[32] GEANT4 Collaboration, GEANT4-a simulation toolkit, Nucl. Instrum. Methods Phys. Res., Sect. A 506, 250 (2003).

[33] S. Ovyn, X. Rouby, and V. Lemaitre, DELPHES, a framework for fast simulation of a generic collider experiment, arXiv: 0903.2225.

[34] DELPHES 3 Collaboration, DELPHES3, A modular framework for fast simulation of a generic collider experiment, J. High Energy Phys. 02 (2014) 057.

[35] J. Alwall et al., A standard format for Les Houches event files, Comput. Phys. Commun. 176, 300 (2007).

[36] N. Desai and P.Z. Skands, Supersymmetry and generic BSM models in PYTHIA8, Eur. Phys. J. C 72, 2238 (2012).

[37] W. Kilian, T. Ohl, J. Reuter, and C. Speckner, QCD in the color-flow representation, J. High Energy Phys. 10 (2012) 022.
[38] T. Ohl, O’Mega: An optimizing matrix element generator, AIP Conf. Proc. 583, 173 (2002).

[39] T. Ohl, O'Mega \& WHIZARD: Monte Carlo event generator generation for future colliders, AIP Conf. Proc. 578, 638 (2001).

[40] P. Stienemeier, S. Braß, P. Bredt, W. Kilian, N. Kreher, T. Ohl et al., WHIZARD3.0: Status and news, in Proceedings of the International Workshop on Future Linear Colliders LCWS2021 (SLAC, 2021), https://www.slac.stanford.edu/ econf/C2103151/.

[41] H. Brooks and P. Skands, Coherent showers in decays of colored resonances, Phys. Rev. D 100, 076006 (2019).

[42] I. Begic, A coherent treatment of qcd radiation from supersymmetric quarks in the antenna shower formalism a coherent treatment of qcd radiation from supersymmetric quarks in the antenna shower formalism, Master's thesis, Monash University, 2019.

[43] N. Desai, F. Domingo, J. S. Kim, R. R. d. A. Bazan, K. Rolbiecki, M. Sonawane et al., Constraining electroweak and strongly charged long-lived particles with CheckMATE, arXiv:2104.04542.

[44] M. Fairbairn, A. C. Kraan, D. A. Milstead, T. Sjostrand, P.Z. Skands, and T. Sloan, Stable massive particles at colliders, Phys. Rep. 438, 1 (2007).

[45] CMS Collaboration, Search for decays of stopped exotic long-lived particles produced in proton-proton collisions at $\sqrt{s}=13 \mathrm{TeV}$, J. High Energy Phys. 05 (2018) 127.

[46] ATLAS Collaboration, Search for heavy charged long-lived particles in the ATLAS detector in $36.1 \mathrm{fb}^{-1}$ of protonproton collision data at $\sqrt{s}=13 \mathrm{TeV}$, Phys. Rev. D 99, 092007 (2019).

[47] ATLAS Collaboration, A search for the decays of stopped long-lived particles at $\sqrt{s}=13 \mathrm{TeV}$ with the ATLAS detector, J. High Energy Phys. 07 (2021) 173.

[48] CMS Collaboration, Search for long-lived charged particles in proton-proton collisions at $\sqrt{s}=13 \mathrm{TeV}$, Phys. Rev. D 94, 112004 (2016).

[49] ATLAS Collaboration, Search for heavy long-lived charged $R$-hadrons with the ATLAS detector in $3.2 \mathrm{fb}^{-1}$ of protonproton collision data at $\sqrt{s}=13 \mathrm{TeV}$, Phys. Lett. B 760, 647 (2016).

[50] CMS Collaboration, Search for fractionally charged particles in $p p$ collisions at $\sqrt{s}=7 \mathrm{TeV}$, Phys. Rev. D 87, 092008 (2013).

[51] G. Busoni, A. De Simone, T. Jacques, E. Morgante, and A. Riotto, Making the most of the relic density for dark matter searches at the LHC $14 \mathrm{TeV}$ run, J. Cosmol. Astropart. Phys. 03 (2015) 022.

[52] A. Albert et al., Recommendations of the LHC Dark Matter Working Group: Comparing LHC searches for dark matter mediators in visible and invisible decay channels and calculations of the thermal relic density, Phys. Dark Universe 26, 100377 (2019).

[53] R. Feger, T. W. Kephart, and R. J. Saskowski, LieART2.0A Mathematica application for Lie algebras and representation theory, Comput. Phys. Commun. 257, 107490 (2020).

[54] S. Coleman, The clebsch-gordan series for su(3), J. Math. Phys. (N.Y.) 5, 1343 (1964). 